Comment. Math. Helv. 75 (2000) 681-700

(C) 2000 Birkhäuser Verlag, Basel

$0010-2571 / 00 / 040681-20 \$ 1.50+0.20 / 0$

Commentarii Mathematici Helvetici

\title{
Symplectic invariants of elliptic fixed points
}

\author{
Karl Friedrich Siburg
}

\begin{abstract}
To the germ of an area-preserving diffeomorphism at an elliptic fixed point, we associate the germ of Mather's minimal action. This yields a strictly convex function which is symplectically invariant and comprises the classical Birkhoff invariants as the Taylor coefficients of its convex conjugate. In addition, however, the minimal action contains information about the local dynamics near the fixed point; for instance, it detects the $C^{0}$-integrability of the diffeomorphism. Applied to the Reeb flow, this leads to new period spectrum invariants for three-dimensional contact manifolds; a particular case is the geodesic flow on a two-dimensional Riemannian manifold, where the period spectrum is the classical length spectrum.
\end{abstract}

Mathematics Subject Classification (2000). 37J, 37E, 53D.

Keywords. Elliptic fixed point, Birkhoff normal form, Aubry-Mather theory, minimal action, Reeb flow, period spectrum, geodesic flow, length spectrum

\section{Introduction}

In this paper, we study the dynamics of an area-preserving diffeomorphism in a neighbourhood of an elliptic fixed point. It is a classical result by G.D. Birkhoff that, under certain nondegeneracy conditions on the linearization, there exists a normal form which is invariant under symplectic coordinate changes. This Birkhoff normal form describes an integrable map whose asymptotics, as one approaches the fixed point, coincide with those of the given non-integrable map. The coefficients of the Taylor polynomial (or series) for the normal form are the so-called Birkhoff invariants.

The goal of this paper is to construct a new "local" symplectic invariant which includes the Birkhoff invariants, but, in contrast, reflects part of the dynamical behaviour near the fixed point. To do so, we introduce a variational principle analogous to that in Aubry-Mather theory for monotone twist maps; namely, we minimize the Lagrangian action over all orbits with a given rotation number. This yields the minimal action, a function canonically associated to the given symplectic map and invariant under symplectic coordinate changes. The key point is to prove that this function is real valued, and hence a nontrivial invariant. Then it follows 
from Aubry-Mather theory that the minimal action contains certain information about the local dynamics near the fixed point. For instance, its differentiability at a rational rotation number is tantamount to the existence of a periodic invariant circle. We will prove the following result; see Theorem 3.2 for the precise formulation.

Theorem. Given an area-preserving diffeomorphism $\phi$ near an elliptic fixed point, the minimal action $\alpha$ is a strictly convex, symplectically invariant function. In addition, one has the following:

1. The Birkhoff invariants are the Taylor coefficients of the convex conjugate $\alpha^{*}$.

2. $\phi$ possesses an invariant circle of rotation number $p / q$, consisting of periodic orbits, if and only if $\alpha$ is differentiable at $p / q$.

3. If $\phi$ has an invariant circle of rotation number $\omega$, its enclosed area is given by $\alpha^{\prime}(\omega)$.

Moreover, in the integrable case, the minimal action is a complete invariant, a fact which is not true for the Birkhoff normal form (unless the map is analytic). We show that the following is true; see Theorem 3.4.

Theorem. Given a symplectic diffeomorphism $\phi$ near an elliptic fixed point, let $\alpha$ denote the associated minimal action. Then the following holds true:

1. If $\phi$ is integrable, $\alpha$ determines $\phi$; in fact, $\alpha^{*}$ is an integrable Hamiltonian (i.e., already in action-angle variables) generating $\phi$.

2. If $\alpha$ is differentiable then $\phi$ is $C^{0}$-integrable.

Symplectic mappings near a fixed point appear often as Poincaré section maps of a closed trajectory. We explain this for the Reeb flow on a contact manifold, a particularly interesting example of which is the geodesic flow. It turns out that the minimal action depends only on the period spectrum of the contact manifold which, in the case of geodesic flows, is the length spectrum from Riemannian geometry. Therefore, the minimal action is a new local length spectrum invariant for compact two-dimensional manifolds, as are all quantities that can be obtained from it (e.g. the Birkhoff invariants).

Minimal orbits - or measures, respectively — play also an important role in other contexts. For planar convex domains, the minimal action is a length spectrum invariant under continuous deformations of the domain [Si3]; this can be seen as a global version of the results presented here. The minimal action appears even in Hofer's geometry of the Hamiltonian diffeomorphism group of cotangent bundles, where it yields a lower bound for the distance of a convex diffeomorphism from the identity [Si1, IS]. A comprehensive exposition of the role of the minimal action in the various contexts is given in [Si4].

Acknowledgement: This work started several years ago with a question of Jürgen Moser while I was still at ETH. He taught me a lot, mathematics and 
more. When I think back to those years it is with deep gratitude.

I am grateful to V. Bangert, G. Knieper, L. Polterovich, and E. Zehnder for helpful discussions during the preparation of this paper, as well as to the anonymous referee for valuable comments.

\section{An estimate in Aubry-Mather theory}

The Principle of Least Action states that, for sufficiently short times, trajectories of a Lagrangian system minimize the action amongst all paths in configuration space with the same end points. If the time interval becomes larger, the EulerLagrange equations describe just critical points of the action functional; they may well be saddle points.

In the eighties, Aubry [Aub] and Mather [Ma1] discovered that monotone twist maps on an annulus possess orbits which minimize the (discrete) action with fixed end points on all time intervals. These minimal orbits turned out to be of crucial importance for a deeper understanding of the complicated orbit structure of monotone twist mappings. In this section, we give a quick review of the relevant theory and prove a perturbation result for the minimal action that we will need later.

Let $\mathbb{S}^{1} \times(a, b) \subset \mathbb{S}^{1} \times \mathbb{R}=T^{*} \mathbb{S}^{1}$ be a plane annulus with $\mathbb{S}^{1}=\mathbb{R} / \mathbb{Z}$, where we allow the cases $a=-\infty$ or $b=+\infty$ (or both). Given a diffeomorphism $\phi$ of $\mathbb{S}^{1} \times(a, b)$ we consider a lift $\widetilde{\phi}$ of $\phi$ to the universal cover $\mathbb{R} \times(a, b)$ of $\mathbb{S}^{1} \times(a, b)$ with coordinates $x, y$. Since $\phi$ is a diffeomorphism so is $\widetilde{\phi}$, and we have $\widetilde{\phi}(x+1, y)=\widetilde{\phi}(x, y)+(1,0)$. In this section, we will always work with (fixed) lifts for which we drop the tilde again and keep the notation $\phi$.

In the case when $a$ or $b$ is finite we assume that $\phi$ extends continuously to $\mathbb{R} \times[a, b]$ by rotations by some fixed angles:

$$
\phi(x, a)=\left(x+\omega_{-}, a\right) \quad \text { resp. } \quad \phi(x, b)=\left(x+\omega_{+}, b\right) .
$$

The numbers $\omega_{ \pm}$are unique after we have fixed the lift. For simplicity, we set $\omega_{ \pm}= \pm \infty$ if $a=-\infty$ or $b=\infty$.

By definition, a (monotone) twist mapping is a $C^{1}$-diffeomorphism

$$
\begin{aligned}
\phi: \mathbb{R} \times(a, b) & \rightarrow \mathbb{R} \times(a, b) \\
\left(x_{0}, y_{0}\right) & \mapsto\left(x_{1}, y_{1}\right)
\end{aligned}
$$

satisfying $\phi\left(x_{0}+1, y_{0}\right)=\phi\left(x_{0}, y_{0}\right)+(1,0)$ as well as the following conditions:

1. $\phi$ preserves orientation and the boundaries of $\mathbb{R} \times(a, b)$, in the sense that $y_{1}\left(x_{0}, y_{0}\right) \rightarrow a, b$ as $y_{0} \rightarrow a, b$

2. if $a$ or $b$ is finite $\phi$ extends to the boundary by a rotation;

3. $\phi$ satisfies a monotone twist condition:

$$
\frac{\partial x_{1}}{\partial y_{0}}>0
$$


4. $\phi$ is exact symplectic; in other words, there is a $C^{2}$-function $h$ such that

$$
y_{1} d x_{1}-y_{0} d x_{0}=d h\left(x_{0}, x_{1}\right) .
$$

The interval $\left(\omega_{-}, \omega_{+}\right) \subset \mathbb{R}$, which can be infinite, is called the twist interval of $\phi$.

\section{Remark 2.1.}

1. The twist condition states that images of verticals are graphs over the $x$-axis. This implies that $\phi$ can be described in the coordinates $x_{0}, x_{1}$ rather than $x_{0}, y_{0}$.

2. The function $h$, which is unique up to an additive constant, is called a generating function for $\phi$ and serves as a discrete version of the Lagrangian action. It is defined on the strip $\left\{(\xi, \eta) \in \mathbb{R}^{2} \mid \omega_{-}<\eta-\xi<\omega_{+}\right\}$and can be extended continuously to its closure. Moreover, it satisfies $\partial_{1} \partial_{2} h<0$ as well as the periodicity condition $h(\xi+1, \eta+1)=h(\xi, \eta)$.

Example 2.2. The simplest example is what is called an integrable twist map which, by definition, preserves the radial coordinate:

$$
\phi\left(x_{0}, y_{0}\right)=\left(x_{0}+f\left(y_{0}\right), y_{0}\right)
$$

with $f^{\prime}>0$. Then $h=h\left(x_{1}-x_{0}\right)$, with $h^{\prime}=f^{-1}$, is strictly convex.

Note that an orbit $\left(\left(x_{i}, y_{i}\right)\right)_{i \in \mathbb{Z}}$ of a monotone twist map $\phi$ is completely determined by the sequence $\left(x_{i}\right)_{i \in \mathbb{Z}}$ via the relations

$$
y_{i}=\partial_{2} h\left(x_{i-1}, x_{i}\right)=-\partial_{1} h\left(x_{i}, x_{i+1}\right) .
$$

Similarly, an arbitrary sequence $\left(\xi_{i}\right)_{i \in \mathbb{Z}}$ corresponds to an orbit of $\phi$ if and only if

$$
\partial_{2} h\left(\xi_{i-1}, \xi_{i}\right)+\partial_{1} h\left(\xi_{i}, \xi_{i+1}\right)=0
$$

for all $i \in \mathbb{Z}$. Thus, on a formal level, orbits may be regarded as "critical points" of the (discrete) action "functional"

$$
\left(\xi_{i}\right)_{i \in \mathbb{Z}} \mapsto \sum_{i \in \mathbb{Z}} h\left(\xi_{i}, \xi_{i+1}\right)
$$

on $\mathbb{R}^{\mathbb{Z}}$. From this point of view, the minimal orbits we are looking for will be "minima" of that "functional".

To make this precise, we call a sequence $\left(x_{i}\right)_{i \in \mathbb{Z}}$ minimal if every finite segment minimizes the action with fixed end points; this means that

$$
\sum_{i=k}^{l-1} h\left(x_{i}, x_{i+1}\right) \leq \sum_{i=k}^{l-1} h\left(\xi_{i}, \xi_{i+1}\right)
$$


for all $\left(\xi_{k}, \ldots, \xi_{l}\right) \in \mathbb{R}^{l-k+1}$ with $\xi_{k}=x_{k}$ and $\xi_{l}=x_{l}$. By (2.1), each minimal sequence $\left(x_{i}\right)_{i \in \mathbb{Z}}$ corresponds to an orbit $\left(\left(x_{i}, y_{i}\right)\right)_{i \in \mathbb{Z}}$; these are called minimal orbits.

The rotation number of an orbit $\left(\left(x_{i}, y_{i}\right)\right)_{i \in \mathbb{Z}}$ of a monotone twist map is given by

$$
\lim _{|i| \rightarrow \infty} \frac{x_{i}}{i}=\lim _{|i| \rightarrow \infty} \frac{x_{i}-x_{0}}{i}
$$

if this limit exists.

The following theorem is the basic result in Aubry-Mather theory. The reader may consult [Ban, Gol, $\mathrm{KH}, \mathrm{MF}$ ] for more details.

Theorem 2.3. ([Aub, Ma1]) A monotone twist map possesses minimal orbits for every rotation number in its twist interval; for rational rotation numbers there are always periodic minimal orbits. Moreover, every minimal orbit lies on a Lipschitz graph over the $x$-axis.

In view of the latter property, minimal orbits resemble invariant circles which, by a classical theorem of Birkhoff, must be Lipschitz graphs (cf. [Si2] and the references therein). By an invariant circle we mean an embedded homotopically nontrivial invariant closed curve in $\mathbb{S}^{1} \times(a, b)$, respectively, its lift to $\mathbb{R} \times(a, b)$.

Remark 2.4.

1. If a monotone twist map possesses an invariant circle of rotation number $\omega$ then every orbit on that circle is minimal [MF, Thm. 17.4].

2. Theorem 2.3 remains true if one considers the more general setting of a monotone twist map on an invariant annulus $\left\{(x, y) \mid u_{-}(x) \leq y \leq u_{+}(x)\right\}$ between the graphs of two functions $u_{ \pm}$; see [MF].

By associating to each $\omega \in\left(\omega_{-}, \omega_{+}\right)$the average action of some (and hence any) minimal orbit $\left(\left(x_{i}, y_{i}\right)\right)_{i \in \mathbb{Z}}$ having rotation number $\omega$, one defines the minimal action

$$
\begin{aligned}
\alpha:\left(\omega_{-}, \omega_{+}\right) & \rightarrow \mathbb{R} \\
\omega & \mapsto \lim _{N \rightarrow \infty} \frac{1}{2 N} \sum_{i=-N}^{N-1} h\left(x_{i}, x_{i+1}\right)
\end{aligned}
$$

\section{Proposition 2.5.}

1. $\alpha$ is strictly convex; in particular, it is continuous.

2. $\alpha$ is differentiable at all irrational numbers.

3. If $\omega=p / q$ is rational, $\alpha$ is differentiable at $p / q$ if and only if there is an invariant circle of rotation number $p / q$ consisting entirely of periodic minimal orbits. 
4. If $\Gamma_{\omega}$ is an invariant circle of rotation number $\omega$ then $\alpha$ is differentiable at $\omega$ with $\alpha^{\prime}(\omega)=\int_{\Gamma_{\omega}} y d x$.

Proof. Everything is well known and can be found in [MF, Ma2], except perhaps for the precise value of $\alpha^{\prime}(\omega)$ in the last part. This follows from Moser's observation that every twist map can be interpolated by a convex Hamiltonian [Mo3], together with [Si1, Thm. 2.1].

We will see now that the minimal action for a perturbation of an integrable twist map is a perturbation of the minimal action for the integrable map. This is made precise in the next theorem; compare [Kat, Lemma 6] for a related argument.

Theorem 2.6. Let $h, h_{0}$ be generating functions for two monotone twist maps such that $h_{0}(s)=c(s-\gamma)^{k}+\mathcal{O}\left((s-\gamma)^{k+1}\right)$ with $c>0, k \geq 2$ generates an integrable twist map, and

$$
h(\xi, \eta)=h_{0}(\eta-\xi)+\mathcal{O}\left((\eta-\xi-\gamma)^{k+m}\right)
$$

as $\eta-\xi \rightarrow \gamma$ with $2 m \in \mathbb{N} \backslash\{0\}$. Then the corresponding minimal actions $\alpha, \alpha_{0}$ satisfy $\alpha_{0}(\omega)=h_{0}(\omega)$ and

$$
\alpha(\omega)=\alpha_{0}(\omega)+\mathcal{O}\left((\omega-\gamma)^{k+m}\right)
$$

as $\omega \rightarrow \gamma$.

Later, we will apply this theorem when $\gamma=\omega_{-}$is the lower boundary point of the twist interval.

Proof. Let us first convince ourselves that $\alpha_{0}=h_{0}$. All orbits of rotation number $\omega$ lie on the invariant circle $\mathbb{S}^{1} \times\left\{\left(h_{0}^{\prime}\right)^{-1}(\omega)\right\}$ and have the same average action $h_{0}(\omega)$. Hence the minimal action $\alpha_{0}(\omega)$ is indeed $h_{0}(\omega)$.

In the following, $C$ always denotes some positive constant that may vary from time to time. Write $h(\xi, \eta)=h_{0}(\eta-\xi)+f(\xi, \eta)$. Since $f$ vanishes up to second order at $\{\eta-\xi=\gamma\}$ we know that minimal orbits of rotation numbers $\omega$ close enough to $\gamma$ are located near $\{\eta-\xi=\gamma\}$ where we have

$$
|f(\xi, \eta)| \leq C h_{0}(\eta-\xi)^{1+\frac{m}{k}} .
$$

Pick a minimal $h$-sequence $\left(x_{i}\right)_{i \in \mathbb{Z}}$ of rotation number $\omega$. The idea is to compare the action of a segment

$$
\left(x_{-N}, x_{-N+1}, \ldots, x_{-1}, x_{0}, x_{1}, \ldots, x_{N-1}, x_{N}\right)
$$

to that of the segment

$$
\left(x_{-N}, x_{0}+(-N+1) \omega, \ldots, x_{0}-\omega, x_{0}, x_{0}+\omega, \ldots, x_{0}+(N-1) \omega, x_{N}\right)
$$


with the same end points. Note that, up to the two end points, the second segment belongs to the minimal $h_{0}$-sequence $\left(x_{0}+i \omega\right)_{i \in \mathbb{Z}}$. The minimality of the sequence $\left(x_{i}\right)_{i \in \mathbb{Z}}$ implies that

$$
\left|x_{i}-x_{0}-i \omega\right| \leq 1
$$

for all $i \in \mathbb{Z}$; see [Ban, Cor. 3.16]. Since $\left(x_{i}\right)_{-N \leq i \leq N}$ minimizes the action we can estimate

$$
\begin{aligned}
& \sum_{i=-N}^{N-1} h\left(x_{i}, x_{i+1}\right) \\
& \leq h\left(x_{-N}, x_{0}+(-N+1) \omega\right)+\sum_{i=-N+1}^{N-2} h\left(x_{0}+i \omega, x_{0}+(i+1) \omega\right) \\
& \quad+h\left(x_{0}+(N-1) \omega, x_{N}\right) \\
& \leq(2 N-2) h_{0}(\omega)+(2 N-2) \max _{\xi}|f(\xi, \xi+\omega)| \\
& +2 \min \left\{\max _{|\eta-\xi| \leq 1+|\omega|}|h(\xi, \eta)|, \max _{\xi, \eta}|h(\xi, \eta)|\right\}
\end{aligned}
$$

where the second inequality uses $h=h_{0}+f$ and (2.3). Dividing this inequality by $2 N$ and taking the limit as $N \rightarrow \infty$ yields

$$
\alpha(\omega) \leq h_{0}(\omega)+\max _{\xi}|f(\xi, \xi+\omega)| \leq \alpha_{0}(\omega)+C|\omega-\gamma|^{k+m}
$$

For the reversed estimate, we obtain from (2.2) that

$$
\sum_{i=-N}^{N-1} h\left(x_{i}, x_{i+1}\right) \geq \sum_{i=-N}^{N-1}\left[h_{0}\left(x_{i+1}-x_{i}\right)-C h_{0}\left(x_{i+1}-x_{i}\right)^{1+\frac{m}{k}}\right] .
$$

Now observe that, because of $k \geq 2$, the function $h_{0}-C h_{0}^{1+\frac{m}{k}}$ is convex near $\gamma$ because $h_{0}$ itself is. This is easily shown by taking the second derivative and comparing the orders in $\omega-\gamma$ for the three different terms. But a convex function $g$ satisfies $g(s)+g(t) \geq 2 g((s+t) / 2)$. Applying this to (2.4) we find

$$
\begin{aligned}
\sum_{i=-N}^{N-1} h\left(x_{i}, x_{i+1}\right) & \geq 2 N\left(h_{0}-C h_{0}^{1+\frac{m}{k}}\right)\left(\frac{\sum_{i=-N}^{N-1} x_{i+1}-x_{i}}{2 N}\right) \\
& =2 N\left(h_{0}-C h_{0}^{1+\frac{m}{k}}\right)\left(\frac{x_{N}-x_{-N}}{2 N}\right) .
\end{aligned}
$$

Again, dividing by $2 N$ and taking the limit yields

$$
\alpha(\omega) \geq h_{0}(\omega)-C h_{0}(\omega)^{1+\frac{m}{k}} \geq \alpha_{0}(\omega)-C|\omega-\gamma|^{k+m},
$$


and the theorem is proven.

Since $\alpha$ is a convex function it possesses a convex conjugate (or Fenchel transform) $\alpha^{*}$ defined by

$$
\alpha^{*}(I)=\max _{\omega}[\omega I-\alpha(\omega)]
$$

Since $\alpha$ is strictly convex, $\alpha^{*}$ is a convex $C^{1}$-function with

$$
\left(\alpha^{*}\right)^{\prime}\left(\alpha^{\prime}(\omega)\right)=\omega
$$

whenever $\alpha^{\prime}(\omega)$ exists [RW, Thm. 11.13]. Flat parts of $\alpha^{*}$ correspond to points of non-differentiability of $\alpha{ }^{1}$

\section{Elliptic fixed points of area-preserving maps}

We consider the germ of a symplectic diffeomorphism at the fixed point $0 \in\left(\mathbb{R}^{2}, \Omega\right)$ where $\Omega$ is some area form on $\mathbb{R}^{2}$. In the following, whenever we pick a representative $\phi: U \rightarrow \mathbb{R}^{2}$, we assume that $U$ is a simply connected neighbourhood of 0 ; this is no loss of generality. Then, by Poincaré's Lemma, the symplectic form $\Omega$ is exact, i.e., there is a $1-$ form $\lambda$ with $\Omega=d \lambda$.

Suppose there is a point $p \in U \backslash\{0\}$ whose iterates $p_{i}=\phi^{i}(p)$ exist for all $i \in \mathbb{Z}$. We want to define the average action and the rotation number of the orbit $\left(p_{i}\right)_{i \in \mathbb{Z}}$. Since $\phi$ is symplectic the $1-$ form $\phi^{*} \lambda-\lambda$ on $U$ is closed, hence exact:

$$
\phi^{*} \lambda-\lambda=d S .
$$

$S$ is called a generating function for $\phi$, and we make it unique by normalizing $S(0)=0 .^{2}$ The average action of the orbit $\left(p_{i}\right)_{i \in \mathbb{Z}}$ with respect to $\lambda$ is defined as

$$
A\left(\left(p_{i}\right)\right)=\lim _{N \rightarrow \infty} \frac{1}{2 N} \sum_{i=-N}^{N-1} S\left(p_{i}\right)
$$

if this limit exists.

We claim that this definition does not depend on the choice of the 1 -form $\lambda$. Indeed, taking another 1 -form $\lambda^{\prime}$ with $d \lambda^{\prime}=d \lambda=\Omega$ the closed 1 -form $\lambda^{\prime}-\lambda$ is

\footnotetext{
1 See [RW] for any question about smooth or non-smooth convex analysis.

2 As an aside, we remark that this normalization is in accordance with setting $H(t, 0)=0$ when $H$ is a Hamiltonian whose flow generates $\phi$ and leaves 0 fixed; for, then the generating function $\int \lambda-H d t$ vanishes at 0 .
} 
exact: $\lambda^{\prime}-\lambda=d F$. The new generating function is given by $S^{\prime}=S+\phi^{*} F-F{ }^{3}$ But the average of $\phi^{*} F$ over an orbit is the same as that of $F$, proving our claim.

Moreover, the average action is invariant under local symplectic coordinate changes $\Phi$ fixing the origin (which are always exact symplectic). The invariance follows from the same argument as above because the generating function for $\Phi \circ \phi \circ \Phi^{-1}$ is given by $\Phi^{*} S+F-\left(\Phi \circ \phi \circ \Phi^{-1}\right)^{*} F$ where we assume that the coordinate change $\Phi$ is generated by $F$.

Next, we want to define the rotation number of an orbit $\left(p_{i}\right)_{i \in \mathbb{Z}}$ in $U \backslash\{0\}$. Roughly speaking, this is its average winding number around the origin. More precisely, we introduce polar coordinates on $\mathbb{R}^{2} \backslash\{0\}=\mathbb{S}^{1} \times(0, \infty)$, and lift $\phi: U \backslash$ $\{0\} \rightarrow \mathbb{R}^{2} \backslash\{0\}$ to a map $\widetilde{\phi}$ which is then defined on some strip in the universal cover $\mathbb{R} \times(0, \infty)$ of $\mathbb{S}^{1} \times(0, \infty)$. Since $\phi$ is an orientation-preserving diffeomorphism, $\widetilde{\phi}$ is a diffeomorphism of degree 1 . Given an orbit $\left(\widetilde{p}_{i}\right)$ of $\widetilde{\phi}$ projecting onto $\left(p_{i}\right)$ and a natural number $N$, we choose a curve $\widetilde{\Gamma}:[-N, N] \rightarrow \mathbb{R} \times(0, \infty)$ with $\widetilde{\Gamma}(i)=\widetilde{p}_{i}$. Call $\Gamma:[-N, N] \rightarrow \mathbb{R}^{2} \backslash\{0\}$ the projection of $\widetilde{\Gamma}$, and close it up to a closed curve $\Gamma_{N}$ by adding a "short" piece (whose lift upstairs lies inside one fundamental domain). Then we define the rotation number of $\left(\widetilde{p_{i}}\right)$ to be

$$
\rho\left(\left(\widetilde{p_{i}}\right), \widetilde{\phi}\right)=\lim _{N \rightarrow \infty} \frac{1}{2 N}\left[\Gamma_{N}\right] \quad \in H_{1}\left(\mathbb{R}^{2} \backslash\{0\}, \mathbb{R}\right)
$$

if this limit exists. Clearly, if we fix the lift $\widetilde{\phi}$, the class $\rho$ does not depend on the particular choice of $\left(\widetilde{p}_{i}\right)$ and $\Gamma_{N}$. Moreover, choosing a different lift $\bar{\phi}$ means adding the class $[\bar{\phi}-\widetilde{\phi}]$ of the deck transformation $\bar{\phi}-\widetilde{\phi}$. Finally, we have a canonical identification $H_{1}\left(\mathbb{R}^{2} \backslash\{0\}, \mathbb{R}\right)=\mathbb{R}$ if we take as generator of $H_{1}\left(\mathbb{R}^{2} \backslash\{0\}, \mathbb{R}\right)$ the class represented by the positively oriented unit circle.

Summarizing, we view the rotation number of an orbit $\left(p_{i}\right)_{i \in \mathbb{Z}}$ in $U \backslash\{0\}$ as a real number $\rho\left(\left(p_{i}\right)\right)=\rho\left(\left(\widetilde{p_{i}}\right), \widetilde{\phi}\right)$, well defined up to integer shifts and invariant under conjugation by homeomorphisms.

Analogous to Aubry-Mather theory, we introduce the following variational principle for the symplectic map $\phi: U \backslash\{0\} \rightarrow \mathbb{R}^{2} \backslash\{0\}$. Having fixed some lift $\widetilde{\phi}$ of $\phi$, we denote by $\alpha(\omega)$ the infimum of average actions of orbits in $U \backslash\{0\}$ with rotation number $\omega$ where, as usual, the infimum over the empty set is $\infty$. This defines the minimal action

$$
\begin{aligned}
\alpha: \mathbb{R} & \rightarrow(-\infty, \infty] \\
\omega & \mapsto \inf \left\{A\left(\left(p_{i}\right)\right) \mid \rho\left(\left(p_{i}\right)\right)=\omega\right\}
\end{aligned}
$$

as a symplectic invariant of the given symplectic germ. Of course, it may be always infinite since it is not clear whether there are any orbits in $U \backslash\{0\}$ at all. Therefore, we have to find situations where the minimal action is a nontrivial invariant.

3 Note that $S^{\prime}$ satisfies our normalization condition $S^{\prime}(0)=0$. 
We make the following assumptions on $\phi$ :

I. $\phi$ is a symplectic $C^{\infty}$-diffeomorphism defined on a simply connected open neighbourhood $U$ of $0 \in\left(\mathbb{R}^{2}, \Omega\right)$ with $\phi(0)=0$;

II. 0 is an elliptic fixed point of $\phi$, i.e., the eigenvalues $\lambda, \bar{\lambda}$ of $D \phi(0)$ lie in $\mathbb{S}^{1} \backslash\{ \pm 1\}$

III. $\lambda$ satisfies the nonresonance condition $\lambda^{k} \neq 1$ for $1 \leq k \leq 4$.

In order to remove the ambiguity in the rotation number, we write $\lambda=e^{2 \pi i a}$ with $0 \leq a<1$ and fix the lift $\widetilde{\phi}$ in such a way that $\widetilde{\phi}(\theta, r) \rightarrow(\theta+a, r)$ as $r \rightarrow 0$. This means that we associate to the fixed point the rotation number $a$ (and not some integer shift of it).

Then, under the assumptions I.-III., there is an analytic symplectic change of coordinates fixing 0 and transforming $\phi$ into a certain normal form in the standard symplectic space $\left(\mathbb{R}^{2}, \Omega_{0}=d x \wedge d y\right)$. In these new coordinates the map $\phi$ takes the form

$$
\begin{gathered}
\left(\begin{array}{l}
x \\
y
\end{array}\right) \mapsto\left(\begin{array}{cc}
\cos 2 \pi \Theta & -\sin 2 \pi \Theta \\
\sin 2 \pi \Theta & \cos 2 \pi \Theta
\end{array}\right)\left(\begin{array}{l}
x \\
y
\end{array}\right)+\mathcal{O}\left(\left(x^{2}+y^{2}\right)^{2}\right) \\
\Theta=a+b\left(x^{2}+y^{2}\right)
\end{gathered}
$$

as $x^{2}+y^{2} \rightarrow 0$. This result goes back to G.D. Birkhoff; a proof can be found in [Mo1]. The leading term, the so-called Birkhoff normal form, is a rotation by an angle $\Theta$ that depends on the radius as long as $b \neq 0$. The numbers $a$ and $b$ are symplectic invariants and called Birkhoff invariants.

Our last assumption is a nonlinearity condition on the Birkhoff normal form: IV. $b \neq 0$, respectively, $b>0$.

If all four conditions I.-IV. are fulfilled we call 0 a general elliptic fixed point of $\phi$.

\section{Remark 3.1.}

1. Without loss of generality we assume $b>0$; the case where $b<0$ can be reduced to that by considering $\phi^{-1}$ instead of $\phi$.

2. The notion of a general elliptic fixed point is intrinsic, i.e., the above conditions are invariant under smooth symplectic coordinate transformations.

Let us call the half-sided germ of a function at a point $x \in \mathbb{R}$ the equivalence class of functions defined on intervals $[x, z)$, where two such functions are equivalent if they agree on some (maybe smaller) interval $[x, y$ ). Finally, from now on, the term invariant circle always means an invariant circle that goes around the fixed point.

The following is the main result in this section. Recall that $\lambda=e^{2 \pi i a}$ with $0 \leq a<1$.

Theorem 3.2. Given the germ of a symplectic diffeomorphism $\phi$ at a general elliptic fixed point, the half-sided germ of the minimal action $\alpha$ at the point $a$ is a nontrivial symplectic invariant. In addition, one has the following: 
1. The Birkhoff invariants are the Taylor coefficients of the convex conjugate $\alpha^{*}$ at 0 .

2. $\phi$ possesses an invariant circle of rotation number $p / q$, consisting of periodic orbits, if and only if $\alpha$ is differentiable at $p / q$.

3. If $\phi$ has an invariant circle of rotation number $\omega$, its enclosed area is given by $\alpha^{\prime}(\omega)$.

Proof. Let $\alpha: \mathbb{R} \rightarrow(-\infty, \infty]$ be the minimal action for $\phi$. We may assume that $\phi$ is already given in the form (3.2); since $\alpha$ is symplectically invariant this does not change anything. To prove that $\alpha$ is nontrivial (i.e. not identically $\infty$ ) we introduce symplectic polar coordinates $(\theta, r) \in \mathbb{S}^{1} \times(0, \infty)$ on $\mathbb{R}^{2} \backslash\{0\}$ by

$$
\left\{\begin{array}{l}
x=\sqrt{2 r} \cos 2 \pi \theta \\
y=\sqrt{2 r} \sin 2 \pi \theta
\end{array}\right.
$$

It is a straightforward calculation to show that

$$
\frac{1}{2}(x d y-y d x)=2 \pi r d \theta
$$

so that the map

$$
\begin{aligned}
\left(\mathbb{R}^{2} \backslash\{0\}, d x \wedge d y\right) & \rightarrow\left(\mathbb{S}^{1} \times(0, \infty), 2 \pi d r \wedge d \theta\right) \\
(x, y) & \mapsto(\theta, r)
\end{aligned}
$$

is exact symplectic with respect to the 1 -forms $1 / 2(x d y-y d x)$ and $2 \pi r d \theta$, respectively. Hence the average action of corresponding orbits stays the same if we pass to $(\theta, r)$-coordinates. The map $\phi$ has the form

$$
\phi:\left(\theta_{0}, r_{0}\right) \mapsto\left(\theta_{1}, r_{1}\right)=\left(\theta_{0}+a+2 b r_{0}, r_{0}\right)+\mathcal{O}\left(r_{0}^{3 / 2}\right)
$$

as $r_{0} \rightarrow 0$. For small enough $r_{0}>0, \phi$ satisfies the monotone twist condition $\partial \theta_{1} / \partial r_{0}=2 b+\mathcal{O}\left(r_{0}^{1 / 2}\right)>0$.

Since $\phi$ is smooth, KAM-theory applies and yields the existence of invariant circles accumulating at the fixed point, respectively the boundary circle $\mathbb{S}^{1} \times\{0\}$; see [Laz, Mo2]. On each of these circles $\Gamma_{\omega}$ the map $\phi$ is conjugated to the rotation by some Diophantine number $\omega$ near $a$; since the twist constant $b$ is positive we have $\omega>a$.

Therefore, perhaps after restriction to a smaller domain, $\phi$ is defined on an invariant annulus in $\mathbb{S}^{1} \times(0, \infty)$ with lower boundary $S^{1} \times\{0\}$. This annulus itself is divided into a sequence of invariant annuli $A_{k}$, approaching $\mathbb{S}^{1} \times\{0\}$ as $k \rightarrow \infty$ and being bounded by KAM-circles $\Gamma_{\omega_{k}^{ \pm}}$with rotation numbers $\omega_{k}^{+}>\omega_{k}^{-}>a$. According to (3.3), the map $\phi$ on each $A_{k} \cup A_{k+1}$ is a smooth monotone twist map whose generating function with $r_{1} d \theta_{1}-r_{0} d \theta_{0}=d h$ is given by

$$
h(\xi, \eta)=\frac{1}{4 b}(\eta-\xi-a)^{2}+\mathcal{O}\left((\eta-\xi-a)^{5 / 2}\right)
$$


as $\eta-\xi \rightarrow a$. The function

$$
h_{0}(s)=\frac{1}{4 b}(s-a)^{2}
$$

describes the integrable twist map $\phi_{0}\left(\theta_{0}, r_{0}\right)=\left(\theta_{0}+a+2 b r_{0}, r_{0}\right)$ approximating $\phi$. Notice that $h$ is normalized according to our convention; namely, $h(\xi, \eta) \rightarrow 0$ as $\eta-\xi \rightarrow a$ which means that the (hypothetical) value of $h$ at the fixed point is 0 .

Now we apply Aubry-Mather theory for $\phi$ on each "double" annulus $A_{k} \cup A_{k+1}$. In view of Theorem 2.3 and Remark 2.4, there are minimal orbits for every rotation number $\omega \in\left(\omega_{k}^{-}, \omega_{k+1}^{+}\right)$. This allows us to define the minimal action $\alpha$ in the sense of Section 2, which is a strictly convex function on the interval $\left(\omega_{k}^{-}, \omega_{k+1}^{+}\right)$.

We claim that this $\alpha$ is the minimal action as defined in (3.1). First of all, the notions of average action and rotation number agree. Therefore, the only thing to check is that the set of orbits over which we minimize is the same in both settings. This follows from the fact that all orbits of rotation numbers $\omega \in\left(\omega_{k}^{-}, \omega_{k+1}^{+}\right)$lie in the annulus $A_{k} \cup A_{k+1}$. Indeed, suppose that a monotone twist map possesses two invariant circles $\Gamma_{\omega^{ \pm}}$of rotation numbers $\omega^{-}<\omega^{+}$. Then, if an orbit lies outside the annulus formed by $\Gamma_{\omega^{-}}$and $\Gamma_{\omega^{+}}$, its rotation number must lie outside $\left(\omega^{-}, \omega^{+}\right)$; this is a simple consequence of the twist property.

Thus, the minimal action $\alpha$ is a real valued, strictly convex function on each interval $\left(\omega_{k}^{-}, \omega_{k+1}^{+}\right)$. Note that the annuli $A_{k} \cup A_{k+1}$ overlap so each rotation number $\omega_{k}^{ \pm}$is an interior point at some stage, and the different pieces of $\alpha$ really fit together. Moreover, as $k \rightarrow \infty$, the rotation numbers $\omega_{k}^{ \pm}$tend to $a$ and the average actions to zero, so that the minimal action extends to a strictly convex function $\alpha:[a, a+\delta) \rightarrow \mathbb{R}$ with $\alpha(a)=0$.

This proves the first part of the theorem. The assertion that the minimal action determines the existence of periodic invariant circles as well as the enclosed areas of invariant circles follows immediately from Proposition 2.5. It remains to prove that the minimal action encodes the Birkhoff invariants. For this, we consider the convex conjugate $\alpha^{*}(I)=\max _{\omega}[\omega I-\alpha(\omega)]$ which is a strictly convex $C^{1}$-function defined on some interval $\left[0, \delta^{*}\right)$. Applying Theorem 2.6, we conclude from (3.4) that

$$
\alpha(\omega)=h_{0}(\omega)+\mathcal{O}\left((\omega-a)^{5 / 2}\right)=\frac{1}{4 b}(\omega-a)^{2}+\mathcal{O}\left((\omega-a)^{5 / 2}\right)
$$

as $\omega \rightarrow a$ which implies an analogous formula for $\alpha^{*}(I)$ as $I \rightarrow 0$ [RW, Ex. 8.8]:

$$
\alpha^{*}(I)=h_{0}^{*}(I)+\mathcal{O}\left(I^{5 / 2}\right)=a I+b I^{2}+\mathcal{O}\left(I^{5 / 2}\right)
$$

Hence the Taylor coefficients of $\alpha^{*}$ at 0 are indeed the Birkhoff invariants $a$ and $b$, and the theorem is completely proven. 
Remark 3.3.

1. Theorem 3.2 shows that the minimal action is a local invariant in the sense that it contains information not just about the asymptotic behaviour of $\phi$ at the fixed point, but also about the dynamics away from it.

2. The assumption that $\phi$ is smooth is not really necessary; in fact, Theorem 3.2 is true for $C^{5}$-diffeomorphisms [Mo2]. For the sake of simplicity, however, we restrict ourselves to the smooth case.

3. If the Birkhoff normal form approximates the given map $\phi$ up to order $\left(x^{2}+\right.$ $\left.y^{2}\right)^{k}$ with $k \geq 2$, then the Taylor coefficients of $\alpha^{*}$ exist up to order $k$ and Theorem 2.6 implies that they are precisely the $k$ Birkhoff invariants of $\phi$.

4. The fact that the Birkhoff invariants are determined by the actions of periodic orbits (via the labelled length spectrum) was first formulated by Colin de Verdiere [CdV2]. The minimal action, respectively its convex conjugate, can be viewed as an extension of the labelled length spectrum from the rational numbers to the reals.

5. The minimal action $\alpha$ may be interpreted as a "partial integral" for the map $\phi$. This goes as follows. Consider the set $\mathcal{M} \subset U \backslash\{0\}$ of minimal orbits. Then the function $p \mapsto \alpha\left(\rho\left(\phi^{i}(p)\right)\right)$ from $\mathcal{M}$ to $\mathbb{R}$ is constant along orbits but not constant everywhere.

In general, the "partial integral" mentioned in the last remark is neither defined in a whole neighbourhood of 0 , nor is it differentiable. In the special situation when $\phi$ possesses a genuine integral, however, the minimal action turns out to be an integral. In this context, recall that a smooth area-preserving map $\phi$ defined near the elliptic fixed point 0 is called integrable if, perhaps after restricting $\phi$ to some smaller neighbourhood $U$ of 0 , there is a smooth fibration of $U \backslash\{0\}$ by invariant circles. $\phi$ is called $C^{0}$-integrable if there is a $C^{0}$-fibration by invariant circles.

Theorem 3.4. Given a symplectic diffeomorphism $\phi$ near a general elliptic fixed point, let $\alpha$ denote the associated minimal action. Then the following holds true:

1. If $\phi$ is integrable, $\alpha^{*}$ is an integrable Hamiltonian generating $\phi$.

2. If $\alpha$ is differentiable then $\phi$ is $C^{0}$-integrable.

Proof. In order to prove the first assertion, we pass to angle-action coordinates $\left(\theta_{0}, I_{0}\right) \in \mathbb{S}^{1} \times(0, \epsilon)$ in which we have $\phi:\left(\theta_{0}, I_{0}\right) \mapsto\left(\theta_{1}, I_{1}\right)=\left(\theta_{0}+H^{\prime}\left(I_{0}\right), I_{0}\right)$ with a smooth strictly convex Hamiltonian $H$. Moreover, $I_{1} d \theta_{1}-I_{0} d \theta_{0}=d S^{*}$ with $S\left(\theta_{0}, I_{1}\right)=\theta_{0} I_{1}+H\left(I_{1}\right)$, which means that $S^{*}=H^{*}$ is a generating function for the integrable twist map $\phi$. Hence $H=\alpha^{*}$ is an autonomous integrable Hamiltonian generating $\phi$.

We show the second assertion. According to Proposition 2.5, the minimal action is differentiable at irrational numbers, and it is differentiable at rationals if and only if there is an invariant circle consisting of (periodic) minimal orbits 
of the corresponding rotation number. Therefore, if $\alpha$ is differentiable we obtain invariant circles for all rotation numbers by taking limits of rational ones, so $\phi$ is $C^{0}$-integrable.

\section{Remark 3.5.}

1. We see that, in the integrable case, the dynamics of $\phi$ are completely determined by the symplectic invariant $\alpha$. This is not true for the Birkhoff normal form unless $\phi$ is analytic; see [Ito].

2. As a strictly convex function, $\alpha$ is differentiable if and only if it is $C^{1}$ [RW, Thm. 11.13].

Finally, we just mention that there are higher order Birkhoff normal forms near an elliptic fixed point if the eigenvalue $\lambda=e^{2 \pi i a}$ at the fixed point satisfies nonresonance conditions of higher order. For instance, if $\lambda$ is not a root of unity the Birkhoff normal form is a formal power series. In general, the coordinate transformation bringing $\phi$ to that normal form will be a divergent power series. We refer to $[\mathrm{SM}]$ for proofs and more details. Everything in this section can also be formulated in this more general context but we forgo such extensions.

\section{Contact flows near an elliptic closed characteristic}

We consider a smooth compact manifold $M$ of odd dimension $2 n+1 \geq 3$ equipped with a contact form $\beta$. By definition, a contact form is a 1-form on $M$ such that $\beta \wedge(d \beta)^{n}$ is a volume form. This means that the kernel of $\beta$ defines a maximally non-integrable hyperplane field in $T M$. The so-called Reeb vector field $X$ is defined by the equations

$$
i_{X} d \beta=0 \quad \text { and } \quad i_{X} \beta=1 .
$$

Periodic trajectories of the Reeb flow are also called closed characteristics.

Example 4.1. If $N$ is a Riemannian manifold then the unit cotangent bundle $T_{1}^{*} N$ is a contact manifold with contact form $\beta=\left.\lambda\right|_{T_{1}^{*} N}$ where $\lambda$ is the Liouville form on $T^{*} N$. The Reeb vector field is the Hamiltonian vector field corresponding to the geodesic flow on $T_{1} N$.

Assume $\gamma$ is a periodic trajectory of (prime) period $T$ of the Reeb flow, and consider a transverse local section $W$ at some point $p \in \gamma$. This is a $2 n$-dimensional manifold, and we equip it with the symplectic form $\omega=i^{*} d \beta$ where $i: W \hookrightarrow M$ is the inclusion. We denote by $\phi$ the Poincaré return map, defined on a small neighbourhood around $p$ which we identify with a small neighbourhood $U$ of $0 \in$ $\mathbb{R}^{2 n} ;$ call $S: U \rightarrow \mathbb{R}$ the first return time. Then $\phi(0)=0$ and $S(0)=T$.

It is well known that $\phi$ is symplectic - this is just a reformulation of the fact that time and energy are conjugate variables in Hamiltonian mechanics. In fact, $\phi$ 
is even exact symplectic as the following observation shows; compare, for instance, [FG, Prop. 2.1].

Lemma 4.2. $\phi$ is exact symplectic with generating function $S$, i.e., $\phi^{*} \beta-\beta=d S$.

Proof. We denote the flow of $X$ by $\psi^{t}$ and consider the family of mappings $f_{t}(z)=$ $\psi^{t S(z)}(z)$. Then $f_{1}=\phi$ and $\dot{f}_{t}(z)=S(z) X\left(f_{t}(z)\right)$. Therefore

$$
\phi^{*} \beta-\beta=\int_{0}^{1} \frac{d}{d t} f_{t}^{*} \beta d t=\int_{0}^{1} f_{t}^{*}\left(i_{\dot{f}_{t}} d \beta+d i_{\dot{f}_{t}} \beta\right) d t=d S
$$

in view of (4.1).

The general question is how much information about the geometry of the contact manifold $M$ is encoded in the closed characteristics. In the following, let us consider a continuous deformation $\beta_{s}, s \in[0,1]$, of contact forms on $M$ such that each $\left(M, \beta_{s}\right)$ has the same period spectrum, i.e. the same set of periods of closed characteristics. Françoise and Guillemin [FG] conjectured that such a deformation must be trivial, if it also fixes the set of (symplectic conjugacy classes of) linearized Poincaré maps. They proved that, if $\gamma_{s}$ is a nondegenerate elliptic closed characteristic without resonances, the Birkhoff invariants of $\gamma_{s}$ stay fixed during the deformation. This was generalized by Popov [Pop] who showed that the Birkhoff invariants as well as the Liouville classes of invariant tori stay fixed, even allowing resonances and dropping the condition that the deformation preserves the linearized Poincaré maps. What we will do is to show that, for the three-dimensional case, there is a stronger invariant than just the Birkhoff normal form, namely the minimal action.

For this, we consider a three-dimensional contact manifold $M$ with a closed characteristic $\gamma$ such that the corresponding Poincaré map $\phi$ has 0 as a general elliptic fixed point (in the terminology of Section 3). This is independent of the choices of the point on $\gamma$ and the transverse section because two Poincaré maps are symplectically conjugated and the conditions I.-IV. stated at the beginning of Section 3 are invariant under such conjugations. We describe this situation by saying that $\gamma$ is a general elliptic closed characteristic. The eigenvalues of $D \phi(0)$ are called the Floquet multipliers of $\gamma$. Then, applying the theory from Section 3, we can associate to $\gamma$ the half-sided germ of the minimal action $\alpha{ }^{4}$ Note that this is independent of the choices of the point on $\gamma$ and the transverse section.

Now let $\beta_{s}, s \in[0,1]$, be a continuous family of contact forms on $M$, all having the same period spectrum, such that there is a continuous family of general ellip-

\footnotetext{
4 To be really consistent with our notation from the previous section where we assumed that generating functions satisfy $S(0)=0$, we replace the first return time $S(\cdot)$ by $S(\cdot)-T$.
} 
tic closed characteristics $\gamma_{s}$. The next proposition states that the corresponding minimal actions $\alpha_{s}$ do not depend on $s$.

Proposition 4.3. Suppose $\beta_{s}, s \in[0,1]$, is a continuous deformation of contact forms preserving the period spectrum, with a continuous family of general elliptic closed characteristics $\gamma_{s}$. Then, as germs, $\alpha_{s}=\alpha_{0}$ for all $s \in[0,1]$.

Proof. Associated to each closed characteristic $\gamma_{s}$, we have the germ of the minimal action $\alpha_{s}$ for the corresponding Poincaré return map. Being continuous, each $\alpha_{s}$ is uniquely defined by its values on $\mathbb{Q}$. We will show below that, for a fixed rational rotation number $p / q$, the values $\alpha_{s}(p / q)$ vary continuously with $s$. Postponing the proof, we claim that these values must be constant. Indeed, the period spectrum - which is independent of $s$ by assumption - has Lebesgue measure 0 in $\mathbb{R}$. This follows from Sard's Theorem since closed characteristics correspond to critical points of a smooth function; see, for instance, [Pop, Prop. 3.2]. Therefore the values $\alpha_{s}(p / q)$ vary continuously in a set of measure zero, so they must stay fixed.

It remains to prove that $\alpha_{s}(p / q)$ is continuous in $s$. For this, we recall from Theorem 2.3 that for rational rotation numbers there is always a periodic minimal orbit. Besides being periodic, these so-called Birkhoff orbits have the additional property that they are ordered as if they were orbits of a rigid rotation, and they can be found by minimizing the (discrete) action on the compact space of ordered periodic sequences [KH, Thm. 9.3.7]. As minima, the corresponding minimal values $\alpha_{s}(p / q)$ are indeed continuous in $s$.

In fact, one can even eliminate the assumption that we are given a family of general elliptic closed characteristics; its existence follows already from the preservation of the period spectrum. Compare [Pop, Lemma 3.5] for a similar argument.

Proposition 4.4. Suppose $\beta_{s}, s \in[0,1]$, is a continuous deformation of contact forms preserving the period spectrum, such that $\beta_{0}$ admits a general elliptic closed characteristic $\gamma_{0}$.

Then there is a continuous family of general elliptic closed characteristics $\gamma_{s}$ for each $\beta_{s}, s \in[0,1]$; moreover, their periods and Floquet multipliers do not depend on $s$.

Proof. First of all, the condition that $\gamma_{0}$ is general guarantees that 1 is not a Floquet multiplier of $\gamma_{0}$. This implies that one can continue the fixed point of the Poincaré map, corresponding to $\gamma_{0}$, uniquely as a fixed point for small $s>0$, corresponding to a periodic trajectory $\gamma_{s}$. Moreover, because everything changes continuously with $s$, the new closed characteristics $\gamma_{s}$ are general elliptic provided $s$ is small enough, say, for $s \in[0, \delta)$. In addition, since the period spectrum has Lebesgue measure 0 , the periods of $\gamma_{s}$ are all the same. 
To each $\gamma_{s}$ we associate the germ of the minimal action $\alpha_{s}$. Proposition $4.3 \mathrm{im}-$ plies $\alpha_{s}=\alpha_{0}$. It follows that the Birkhoff invariants of the Poincaré map — which are the Taylor coefficients of $\alpha_{s}^{*}$ (Theorem 3.2) — do not change along the deformation. In particular, the Floquet multipliers stay fixed during the deformation. This proves the assertion for $s \in[0, \delta)$.

Taking limits of the closed characteristics $\gamma_{s}$ as $s \rightarrow \delta$, we find a closed characteristic for $s=\delta$. Moreover, the Poincaré maps of $\gamma_{s}$ converge in the $C^{\infty}$-topology to the Poincaré map of $\gamma_{\delta}$. Our assumption that the period spectrum remains unchanged implies that $\gamma_{\delta}$ satisfies the conditions I.-III. from Section 3. On the other hand, we know that the Birkhoff invariants of $\gamma_{\delta}$ are the same as those of $\gamma_{0}$. Thus, $\gamma_{\delta}$ is again a general elliptic closed characteristic.

This proves that the set of parameters $s$, for which there is a continuous family of general elliptic closed characteristics, beginning with $\gamma_{0}$, is open and closed in $[0,1]$. This finishes the proof of the proposition.

Now we can translate our results for fixed points of symplectic mappings into the language of contact geometry. The key point is that the minimal action is a period spectrum invariant under continuous deformations of the contact form.

Theorem 4.5. Suppose $\beta_{s}, s \in[0,1]$, is a continuous family of contact forms on a three-dimensional manifold that preserves the period spectrum, such that $\beta_{0}$ admits a general elliptic closed characteristic $\gamma_{0}$.

Then there is a continuous family of general elliptic closed characteristics $\gamma_{s}$ whose half-sided germs of minimal actions do not depend on $s$. In particular, this implies the following:

1. The Birkhoff invariants of $\gamma_{0}$ and $\gamma_{1}$ are the same.

2. The Poincaré map $\phi_{1}$ possesses an invariant circle of rotation number $p / q$, consisting of periodic orbits, if and only if $\phi_{0}$ does.

3. If $\phi_{0}$ and $\phi_{1}$ each have an invariant circle of rotation number $\omega$, their enclosed areas agree.

4. If $\phi_{0}$ is integrable then $\phi_{1}$ is $C^{0}$-integrable.

Proof. Proposition 4.4 implies that we have a family of minimal actions $\alpha_{s}$ which, by Proposition 4.3, are all equal. Thus, the (half-sided germ of the) minimal action $\alpha=\alpha_{0}$ is a period spectrum invariant.

We prove the four implications. Since the Birkhoff invariants are the Taylor coefficients of $\alpha^{*}$ (Theorem 3.2), they are invariant too. Moreover, $\phi_{1}$ possesses a periodic invariant circle of rotation number $p / q$ if and only if $\alpha$ is differentiable at $p / q$; since $\alpha$ is invariant, the same holds true for $\phi_{0}$. A similar argument proves the third statement because the area enclosed by an invariant circle is given by $\alpha^{\prime}(\omega)$. Finally, if $\phi_{0}$ is integrable then $\alpha^{*}$ is an integrable Hamiltonian (Theorem 3.4); in particular, $\alpha$ is smooth, which implies the $C^{0}$-integrability of $\phi_{1}$. 


\section{Geodesic flows near an elliptic closed geodesic}

The geodesic flow on the unit tangent bundle of a smooth compact Riemannian manifold $N$, respectively its conjugate Hamiltonian flow on $T_{1}^{*} N$, is a particular example of a Reeb flow (Example 4.1). Therefore, all statements from the previous section apply to geodesic flows on surfaces, where the period spectrum becomes the classical length spectrum of $N$, i.e. the set of lengths of closed geodesics.

More precisely, let $g_{s}, s \in[0,1]$, be a continuous family of Riemannian metrics on a surface preserving the length spectrum. Assume that $g_{0}$ possesses a general elliptic closed geodesic $\gamma_{0}$. Then, according to Proposition 4.4, we have a family of general elliptic closed geodesics $\gamma_{s}$ for $g_{s}$, and, associated to it, the half-sided germs of their minimal actions $\alpha_{s}$.

The next result is the analogue of Proposition 4.3.

Proposition 5.1. Under the above assumptions, one has $\alpha_{0}=\alpha_{1}$, i.e., the minimal action is a length spectrum invariant under continuous deformations of the metric.

We may formulate a more pointed version of this as

"Invariance Principle". Every quantity, geometric or not, that can be expressed in terms of $\alpha$ is a length spectrum invariant under continuous deformations of the metric.

Now, for geodesic flows, Theorem 4.5 translates as follows.

Theorem 5.2. Suppose $g_{s}, s \in[0,1]$, is a continuous deformation of Riemannian metrics on a two-dimensional manifold that preserves the length spectrum, such that $g_{0}$ admits a general elliptic closed geodesic $\gamma_{0}$.

Then there is a continuous family of general elliptic closed geodesics $\gamma_{s}$ whose half-sided germs of minimal actions do not depend on s. In particular, this implies the following:

1. The Birkhoff invariants of $\gamma_{0}$ and $\gamma_{1}$ are the same.

2. The Poincaré map $\phi_{1}$ possesses an invariant circle of rotation number $p / q$, consisting of periodic orbits, if and only if $\phi_{0}$ does.

3. If $\phi_{0}$ and $\phi_{1}$ each have an invariant circle of rotation number $\omega$, their enclosed areas agree.

4. If $\phi_{0}$ is integrable then $\phi_{1}$ is $C^{0}$-integrable. ${ }^{5}$

\footnotetext{
5 Steve Zelditch informed me that Giovanni Forni and he had proven the following stronger version independently; see the announcement in [Zel1]. Suppose you are given an analytic, rotationally symmetric metric $g$ on $S^{2}$ with certain nondegeneracy conditions; in this case, the geodesic flow of $g$ is completely integrable. Then, if $h$ is another metric with the same Laplace spectrum as $g$, the geodesic flow of $h$ is completely $C^{0}$-integrable.
} 
The problem to decide whether the length, respectively Laplace, spectrum characterizes a manifold up to isometries (and in which class of metrics) is central in Riemannian geometry. For instance, Kac' famous question "Can one hear the shape of a drum?" asked whether there are non-isometric domains in the plane that have the same Laplace spectrum; it is well known that the answer is yes. The Laplace spectrum is related to the length spectrum via trace formulae and Poisson relations; we refer to [CdV1, GM, Zel1] for details and more references. Zelditch [Zel2] showed that a special class of real analytic surfaces of revolution is completely determined by the Laplace spectrum. The Birkhoff normal form is still an essential ingredient for the proof but does not suffice to obtain the full result.

\section{References}

[Aub] S. Aubry, The twist map, the extended Frenkel-Kontorova model and the devil's staircase, Physica 7D (1983), 240-258.

[Ban] V. Bangert, Mather sets for twist maps and geodesics on tori, in: Kirchgraber, Walther (eds.): Dynamics Reported 1, 1-56, J. Wiley \& Sons and B.G. Teubner 1988.

[CdV1] Y. Colin de Verdière: Spectre du Laplacien et longueurs des géodésiques périodiques I, Comp. Math. 27 (1973), 83-106.

[CdV2] Y. Colin de Verdière, Sur les longueurs des trajectoires périodiques d'un billard, in: Dazord, Desolneux (eds.): Géométrie symplectique et de contact, Sem. Sud-Rhod. Géom. (1984), 122-139.

[FG] J.P. Françoise, V. Guillemin, On the period spectrum of a symplectic mapping, J. Funct. Anal. 100 (1991), 317-358.

[GM] V. Guillemin, R. Melrose, The Poisson summation formula for manifolds with boundary, Adv. Math. 32 (1979), 204-232.

[Gol] C. Golé, A new proof of the Aubry-Mather's theorem, Math. Z. 210 (1992), 441-448.

[Ito] H. Ito, Convergence of Birkhoff normal forms for integrable systems, Comment. Math. Helv. 64 (1989), 412-461.

[IS] R. Iturriaga, H. Sánchez-Morgado: A minimax selector for a class of Hamiltonians on cotangent bundles, preprint.

[Kat] A. Katok, Minimal orbits for small perturbations of completely integrable Hamiltonian systems, in: McGehee, Meyer (eds.): Twist Mappings and Their Applications, IMA Volumes in Mathematics and its Applications 44, Springer-Verlag, 1992.

$[\mathrm{KH}]$ A. Katok, B. Hasselblatt, Introduction to the Modern Theory of Dynamical Systems, Cambridge University Press, 1995.

[Laz] V. Lazutkin, KAM Theory and Semiclassical Approximations to Eigenfunctions, SpringerVerlag, 1993.

[Ma1] J.N. Mather, Existence of quasi-periodic orbits for twist homeomorphisms of the annulus, Topology 21 (1982), 457-467.

[Ma2] J.N. Mather, Differentiability of the minimal average action as a function of the rotation number, Bol. Soc. Bras. Mat., Nova Ser. 21 (1990), 59-70.

[Ma3] J.N. Mather, Action minimizing invariant measures for positive definite Lagrangian systems, Math. Z. 207 (1991), 169-207.

[MF] J.N. Mather, G. Forni, Action minimizing orbits in Hamiltonian systems, in: Graffi (ed.): Transition to Chaos in Classical and Quantum Mechanics, Springer LNM 1589 (1992), 92-186.

[Mo1] J. Moser, Proof of a generalized form of a fixed point theorem due to G.D. Birkhoff, in: 
do Carmo, Palis (eds.): Geometry and Topology, Springer LNM 597 (1977), 464-494.

[Mo2] J. Moser, Stable and random motion in dynamical systems, Princeton University Press, 1977.

[Mo3] J. Moser, Monotone twist mappings and the calculus of variations, Ergod. Th. ES Dynam. Sys. 6 (1986), 401-413.

[Pop] G. Popov, Length spectrum invariants of Riemannian manifolds, Math. Z. 213 (1993), 311-351.

[RW] R.T. Rockafellar, R.J.-B. Wets, Variational Analysis, Springer-Verlag, 1998.

[Si1] K.F. Siburg, Action minimizing measures and the geometry of the Hamiltonian diffeomorphism group, Duke Math. J. 92 (1998), 295-319.

[Si2] K.F. Siburg, A dynamical systems approach to Birkhoff's Theorem, Enseign. Math. 44 (1998), 291-303.

[Si3] K.F. Siburg, Aubry-Mather theory and the inverse spectral problem for planar convex domains, Israel J. Math. 113 (1999), 285-304.

[Si4] K.F. Siburg, The minimal action in geometry and dynamics, preprint.

[SM] C.L. Siegel, J. Moser, Lectures on Celestial Mechanics, Springer-Verlag, 1971.

[Zel1] S. Zelditch, Normal forms and inverse spectral theory, Journées Équations aux dérivées partielles, 2-5 June 1998, GDR 1151 (CNRS).

[Zel2] S. Zelditch: The inverse spectral problem for surfaces of revolution, J. Diff. Geom. 49 (1998), 207-264.

Karl Friedrich Siburg

Fakultät für Mathematik

Ruhr-Universität Bochum

D-44780 Bochum

Germany

e-mail: siburg@math.ruhr-uni-bochum.de

(Received: January 31, 2000) 